\title{
RECONSTRUÇÃO DO LIGAMENTO CRUZADO ANTERIOR COM O TERÇO CENTRAL DO TENDÃO DO MÚSCULO QUADRÍCEPS: ANÁLISE DE RESULTADOS APÓS 10 ANOS
}

\author{
RECONSTRUCTION OF THE ANTERIOR CRUCIATE LIGAMENT WITH THE CENTRAL \\ THIRD OF THE QUADRICEPS MUSCLE TENDON: ANALYSIS OF 10-YEAR RESULTS
}

Marcus Valladares Guimarães ', Lúcio Honório de Carvalho Junior², Dalton Lopes Terra ${ }^{3}$

\section{RESUMO}

Objetivo: Avaliar os resultados clínicos utilizando dois diferentes protocolos, 10 anos após a cirurgia de reconstrução do LCA(Ligamento Cruzado Anterior) com o terço central do tendão do músculo quadríceps (TQ). Método: Entre novembro/1997 e abril/1998, 25 pacientes foram submetidos a 25 reconstruções do LCA com TQ pela técnica transtibial. A parte óssea do enxerto foi fixada no túnel femoral com parafuso de interferência e a parte tendinosa no túnel tibial, com parafuso em poste com arruela. Dois pacientes romperam o novo ligamento em entorses durante futebol. Seis não foram encontrados para reavaliação (24\%). Foram avaliados 17 pacientes, sendo 15 homens e duas mulheres, com média de idade na cirurgia de 28,53 $\pm 6,64$ anos. Todos foram examinados com seis meses, um ano e dez anos de cirurgia. A avaliação clínica foi realizada com a escala de Lysholm e a do o joelho, com a do Hospital for Special Surgery. Resultados: As lesões foram operadas após 9,87 $\pm 14,42$ meses do acidente. Segundo a escala de Lysholm, os resultados ao fim do primeiro ano foram de 98,71 $\pm 2,47$ e, ao fim de dez anos, de 97,35 $\pm 3,12$. Usando a escala do Hospital for Special Surgery, a pontuação foi de 95,07 \pm 5,23 com um ano e de $94,87 \pm 4,16$ após 10 anos. Todos os pacientes retornaram ao trabalho nas mesmas condições. Quinze $(88,24 \%)$ retornaram ao mesmo esporte, um com modificação na prática e outro mudou de esporte. Nenhum paciente se queixou dor na área doadora do enxerto após quatro semanas da cirurgia até a última avaliação. Quatro pacientes sofreram ruptura do LCA contralateral em atividades esportivas, sendo três entorses no futebol e uma durante dança. Conclusão: O enxerto do TQ é boa opção para a reconstrução do LCA, mesmo quando decorridos 10 anos do procedimento cirúrgico. Não houve dor na área doadora do enxerto a médio e longo prazo. A taxa de retorno ao esporte foi excelente e não houve alteração da articulação femoropatelar.

Descritores - Joelho; Ligamento cruzado anterior; Músculo quadríceps; Músculo esquelético

\section{ABSTRACT}

Objective: Assess clinical results using two different protocols, 10 years after ACL reconstruction surgery with the central third of quadriceps muscle tendon (QT). Method: Between November 1997 and April/1998, 25 patients were submitted to 25 ACL reconstructions with QT by transtibial technique. The bone portion of the graft was fixated on femoral tunnel with interference screw and the tendinous portion of tibial tunnel with screw with washer. Two patients injured the new when playing soccer. Six patients were not available for follow-up (24\%). Seventeen patients were evaluated, 15 men and two women, with mean age at surgery time of 28.53 \pm 6.64 years. All patients were examined at six months, one year, and ten years after surgery. Clinical evaluation was made by the Lysholm scale, and the knee evaluation, with the Hospital for Special Surgery scale. Results: The patients had their injuries operated after $9.87 \pm 14.42$ months of the accident. According to Lysholm scale, the results at the end of the first year were $98.71 \pm 2.47$ and, after 10 years, $97.35 \pm$ 3.12. Using the Hospital for Special Surgery scale, the mean score was $95.07 \pm 5.23$ in one year, and $94.87 \pm 4.16$ in 10 years. All patients returned to their professional activities with the same previous status. Fifteen (88.24\%) patients were able to return to their sports activities, one by modifying the practice, while another one switched to another sport. No patient complained of pain on the donor area in the medium and long term. The sports return rate was excellent, and no changes were found on the femoropatellar joint.

Keywords - Knee; Anterior cruciate ligament; Quadriceps muscle; Muscle skeletal

1 - Mestre em Medicina pela Universidade Federal de Minas Gerais, Membro do Núcleo de Ortopedia e Traumatologia de Belo Horizonte.

2 - Professor Adjunto do Departamento de Aparelho Locomotor da Faculdade de Medicina da Universidade Federal de Minas Gerais, Doutor em Medicina pela Unifesp.

3 - Membro do Núcleo de Ortopedia e Traumatologia de Belo Horizonte.

Trabalho realizado no Núcleo de Ortopedia e Traumatologia de Belo Horizonte/MG.

Correspondência: Avenida Raja Gabaglia, 2.636 - 30350-540 - Belo Horizonte, MG. Tel.: (31) 3349-2600. Fax: (31) 3349-2700. E-mail: marcusvg@mac.com 


\section{INTRODUÇÃO}

Durante a última década, a cirurgia para reconstrução do LCA tornou-se uma das mais frequentes na Ortopedia. O sucesso em longo prazo é confirmado através de taxas de bons e excelentes resultados, que variam de $75 \%$ a $95 \%$, considerando estabilidade, alívio de sintomas e retorno ao esporte ${ }^{(1)}$.

Sachs et $a l^{(2)}$ mostraram, como complicações mais frequentes da reconstrução do LCA, perda da força do quadríceps, déficit de extensão, dor na face anterior do joelho. A incidência desses problemas era maior quando usado o tendão patelar (TP), considerado "padrão ouro" para reconstrução do LCA. Em $60,8 \%$ dos casos ocorreu déficit de força do quadríceps após um ano de reconstrução do LCA com TP. Embora raras, complicações como fratura de patela e ruptura do TP, crepitação, tendinite e diminuição do movimento patelar foram relatadas, achados confirmados por outros autores ${ }^{(3,4)}$. Em 1975, Cho $^{(5)}$ descreveu a reconstrução do LCA usando o tendão semimembranoso intra-articular. Mais tarde foi associado o gracilis (TF). Os defensores dessa técnica relatam menor incidência de dor na face anterior do joelho, menor frequência de tendinite patelar, menor fraqueza do quadríceps com diminuição do risco de comprometimento da amplitude de movimento (ADM) do joelho ${ }^{(2)}$. As preocupações quanto à resistência e maior elasticidade desses tendões em relação ao LCA, associadas à dificuldade de fixação e diminuição da força de flexão do joelho, não foram comprovadas ao longo dos $\operatorname{anos}^{(6)}$. O uso do tendão do quadríceps (TQ) foi popularizado por Marshall et $a^{(7)}$, mas com resultados iniciais pouco promissores. Fulkerson e Langeland ${ }^{(8)}$, Howe et $a l^{(9)}$ e Staubli et $a l^{(10)}$, e Staubli e Jakob ${ }^{(11)}$, com trabalhos mais consistentes, mostraram que o TQ tem tamanho e resistência adequados para substituir o LCA com menor morbidade do sítio doador. Hoje, o TQ está se tornando mais popular ${ }^{(12)}$.

O objetivo deste estudo é avaliar os resultados clínicos, utilizando dois diferentes protocolos, 10 anos após a cirurgia, de pacientes que foram submetidos à reconstrução do LCA com TQ.

\section{METODOLOGIA}

Entre novembro/1997 e abril/1998, 25 pacientes foram submetidos a 25 reconstruções do LCA com TQ acrescido de fragmento ósseo da patela pela técnica transtibial. A parte óssea do enxerto foi fixada no túnel femoral com parafuso de interferência metálico e a parte tendinosa, no túnel tibial com dois fios de sutura Ethibond \#2 e parafuso de esponjosa com arruela (poste). A técnica cirúrgica e a reabilitação já foram previamente descritas ${ }^{(13)}$.

Seis pacientes foram perdidos durante o seguimento. Foram excluídos dois pacientes que romperam o enxerto do TQ em entorses durante a prática do futebol, três e seis anos após a cirurgia, e foram reoperados. O grupo estudado apresentava 17 pacientes. Todos foram examinados com seis meses, um ano e 10 anos após a cirurgia. A avaliação clínica foi realizada com a escala de Lysholm e a do Hospital for Special Surgery para o joelho.

Dos 17 pacientes, havia 15 homens e duas mulheres (Tabela 1), com média de idade na cirurgia de 28,53 \pm 6,64 anos. As lesões foram operadas após 9,87 $\pm 14,42$ meses da lesão. Duas lesões foram causadas por queda de motocicleta, uma por queda com entorse da própria altura e 13 por entorses no futebol. Um paciente apresentou uma ressecção prévia do coto do LCA por artroscopia. No peroperatório, foram encontrados seis lesões do menisco medial e uma lesão condral femoral medial com $1 \mathrm{~cm}^{(2)}$. Não foi encontrada lesão da articulação femoropatelar. Foram realizadas uma menis-

Tabela 1 - Caracterização do grupo de pacientes.

\begin{tabular}{|c|c|c|}
\hline Número de pacientes & & \\
\hline \multirow{2}{*}{ Sexo } & Masculino & 15 \\
\hline & Feminino & 2 \\
\hline \multirow{2}{*}{ Lado da lesão } & Direito & 9 \\
\hline & Esquerdo & 8 \\
\hline \multicolumn{2}{|c|}{ Idade na cirurgia (anos) } & 28,53 \\
\hline \multicolumn{2}{|c|}{ Tempo entre lesão- cirurgia (meses) } & 6,64 \\
\hline \multicolumn{2}{|c|}{ Tempo entre cirurgia-avaliação (anos) } & 10 \\
\hline \multirow{4}{*}{ Lesão associada } & Lesão condral & 2 \\
\hline & Menisco medial & 7 \\
\hline & Ligamento Colateral Medial (não abordado) & 0 \\
\hline & Menisco lateral & 0 \\
\hline \multirow{5}{*}{ Tipo de Trauma } & Entorse & 13 \\
\hline & Hiperextensão & 0 \\
\hline & Queda de Esqui (neve) & 1 \\
\hline & Queda da própria altura & 1 \\
\hline & Queda de motocicleta em baixa velocidade & 2 \\
\hline Cirurgia pré-reconstrução & Ressecção de coto do LCA & 1 \\
\hline \multirow{4}{*}{ Cirurgia pós-reconstrução } & Liberação do retináculo (artroscópico) & 1 \\
\hline & Meniscectomia lateral parcial & 1 \\
\hline & osteossíntese patela & 1 \\
\hline & Meniscectomia medial & 3 \\
\hline
\end{tabular}

Fonte: Núcleo de Ortopedia e Traumatologia de Belo Horizonte. 
cectomia total e cinco parciais. Posteriormente, foram realizadas uma osteossíntese de patela para tratamento de fratura ocorrida em queda após seis meses de cirurgia, uma liberação artroscópica do joelho para tratamento de artrofibrose, quatro meniscectomias, sendo duas totais e duas parciais, e uma retirada do parafuso em poste na tíbia.

Foi realizado exame radiológico com apoio, em três incidências, dos joelhos operados após 10 anos de seguimento para avaliação da existência ou não de osteoartrose (Figuras 1, 2 e 3).

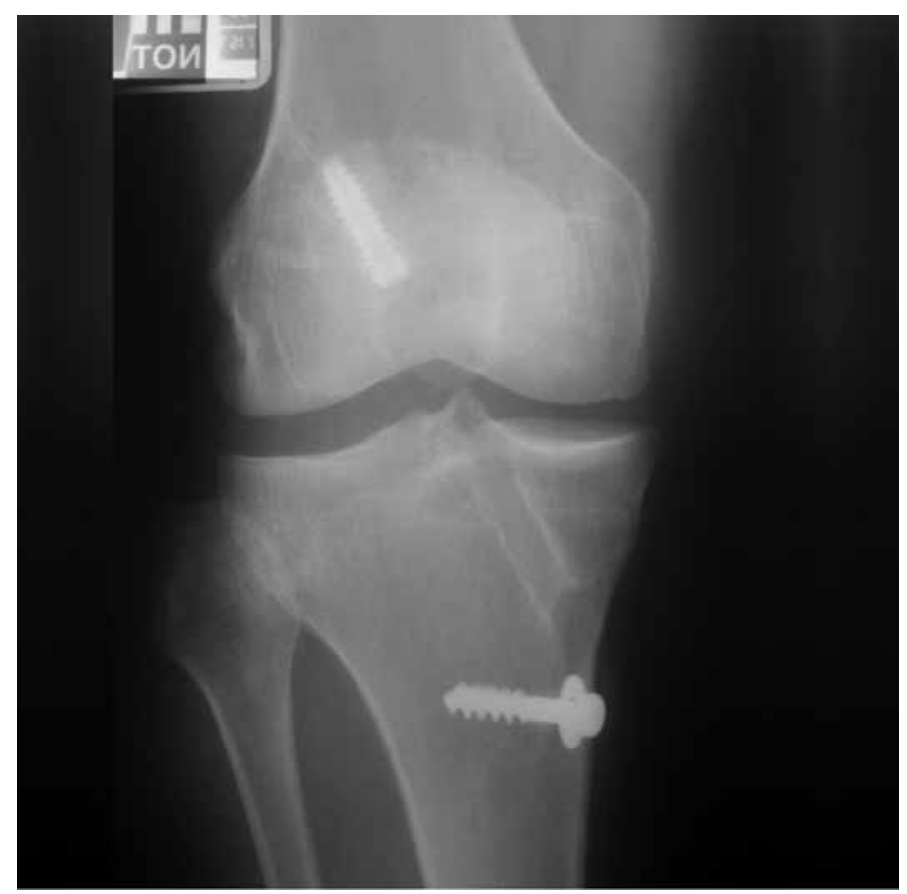

Figura 1 - Radiografia em AP com apoio monopodálico realizada 10 anos após a reconstrução do LCA

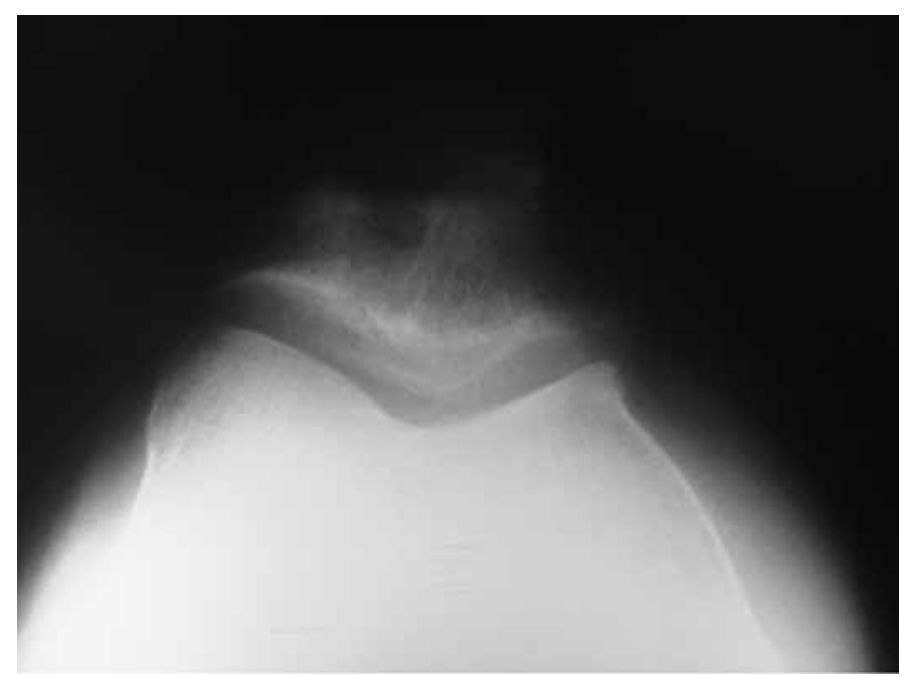

Figura 2 - Radiografia em axial de patela a $30^{\circ}$ de flexão do joelho realizada 10 anos após a reconstrução do LCA

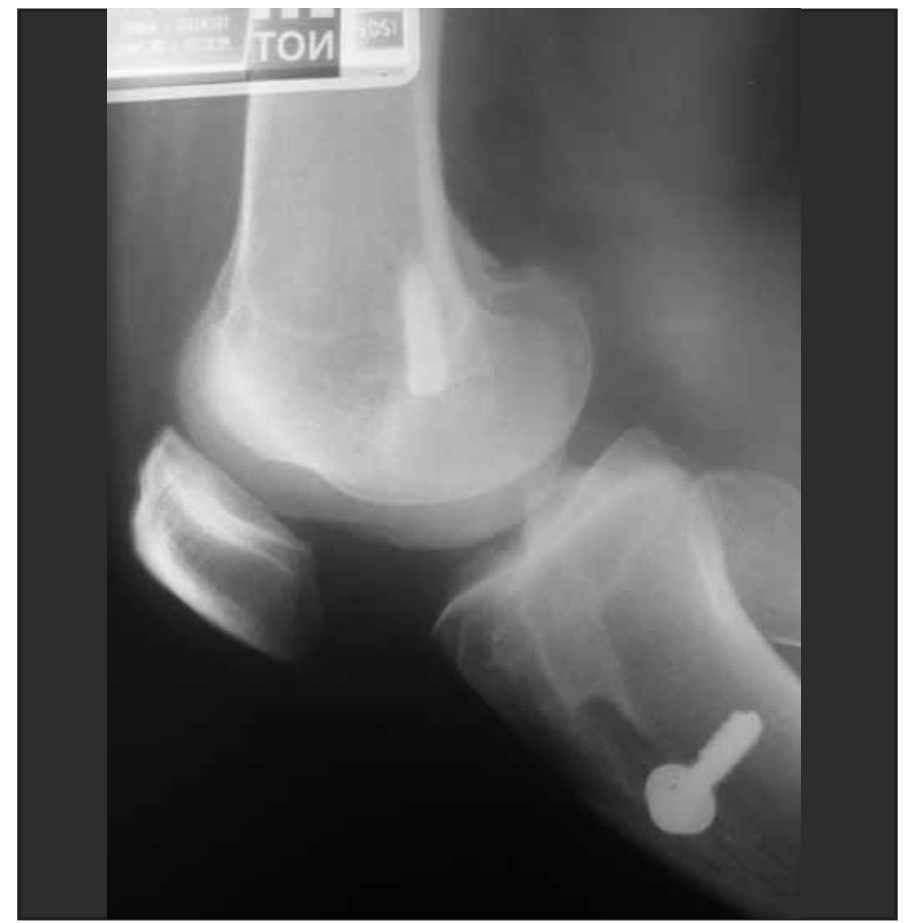

Figura 3 - Radiografia em perfil do joelho sem carga realizada após 10 anos de reconstrução do LCA

\section{RESULTADOS}

Segundo a escala de Lysholm (Tabela 2), o resultado ao fim do primeiro ano foi de $98,71 \pm 2,47$ e de 97,35 $\pm 3,12$ ao fim de dez anos. Usando a escala do Hospital for Special Surgery (HSS) (Tabela 3), a pontuação foi de 95,07 $\pm 5,23$ com um ano e de 94,87 \pm 4,16 após 10 anos (Tabela 4). As queixas que levaram a maior perda de pontos ao final de dez anos na escala de Lysholm foram dor inconstante e leve no esporte $(17,65 \%$ - três pacientes $)$, derrame após esporte intenso $(11,76 \%$ - dois pacientes), dificuldade leve ao subir escadas $(17,65 \%$ - três pacientes), dificuldade leve e ocasional ao agachar (23,53\% - quatro pacientes).

$\mathrm{Na}$ avaliação HSS, todos os pacientes retornaram ao trabalho mantendo as mesmas condições de antes do trauma. Quinze $(88,24 \%)$ pacientes retornaram ao mesmo esporte, um com modificação na sua prática e outro mudou de esporte. Dois pacientes submetidos a meniscectomias mediais totais desenvolveram osteoartrose femorotibial medial e apresentavam limitação da ADM. Dos pacientes avaliados, 16 apresentavam diâmetro da coxa menor de $2 \mathrm{~cm}$ comparando com a outra. $\mathrm{O}$ teste de Lachman foi negativo em 13 pacientes $(76,47 \%)$ e $+/++++$ em quatro. A gaveta anterior foi negativa em 12 pacientes $(70,59 \%) \mathrm{e}+/++++$ em cinco. O pivot test foi negativo em 13 pacientes $(76,47 \%)$ e leve sem movimento em quatro. 
Tabela 2 - Resultados da escala de avaliação de Lysholm

\section{LYSHOLM}

\begin{tabular}{|c|c|c|c|}
\hline Pergunta & Resposta & Pacientes & $\%$ \\
\hline \multirow{3}{*}{$\begin{array}{l}\text { O paciente } \\
\text { manca } \\
\text { (5 pontos) }\end{array}$} & Não (5) & 16 & 94,12 \\
\hline & Levemente ou periodicamente (3) & 1 & 5,88 \\
\hline & Intensamente ou constantemente $(0)$ & & 0,00 \\
\hline \multirow{3}{*}{$\begin{array}{l}\text { Usa algum } \\
\text { suporte } \\
\text { (5 pontos) }\end{array}$} & Não - 5 & 17 & 100,00 \\
\hline & Muleta ou bengala (2) & & 0,00 \\
\hline & Descarga de peso impossivel (0) & & 0,00 \\
\hline \multirow{5}{*}{$\begin{array}{l}\text { Há bloqueio } \\
\text { (15 pontos) }\end{array}$} & Não (15) & 16 & 94,12 \\
\hline & Sensação de restrição sem bloqueio (10) & 1 & 5,88 \\
\hline & Bloqueio ocasional (6) & & 0,00 \\
\hline & Bloqueio frequente (2) & & 0,00 \\
\hline & Joelho bloqueado no exame (0) & & 0,00 \\
\hline \multirow{6}{*}{$\begin{array}{l}\text { Instabilidade } \\
\text { (25 pontos) }\end{array}$} & Nunca (25) & 17 & 100,00 \\
\hline & Raramente durante exercícios severos (20) & & 0,00 \\
\hline & Frequentemente durante exercícios severos (15) & & 0,00 \\
\hline & Ocasionalmente em atividades diárias (10) & & 0,00 \\
\hline & Frequentemente em atividades diárias (5) & & 0,00 \\
\hline & A todo momento $(0)$ & & 0,00 \\
\hline \multirow{6}{*}{$\begin{array}{l}\text { Dor } \\
\text { (25 pontos) }\end{array}$} & Não (25) & 14 & 82,35 \\
\hline & Inconstante ou leve durante exercícios severos (20) & 3 & 17,65 \\
\hline & Forte durante exercícios severos (15) & & 0,00 \\
\hline & Forte depois de caminhar mais de $2 \mathrm{~km}(10)$ & & 0,00 \\
\hline & Forte depois de caminhar menos de $2 \mathrm{~km}(5)$ & & 0,00 \\
\hline & Constante $(0)$ & & 0,00 \\
\hline \multirow{4}{*}{$\begin{array}{l}\text { Derrame } \\
\text { (10 pontos) }\end{array}$} & Não (10) & 15 & 88,24 \\
\hline & Com exercícios severos (6) & 2 & 11,76 \\
\hline & Com exercícios ordinários (2) & & 0,00 \\
\hline & Constante (0) & & 0,00 \\
\hline \multirow{4}{*}{$\begin{array}{l}\text { Subir escadas } \\
\text { (10 pontos) }\end{array}$} & Sem problemas (10) & 14 & 82,35 \\
\hline & Leve dificuldade (6) & 3 & 17,65 \\
\hline & Um degrau por vez (2) & & 0,00 \\
\hline & impossível (0) & & 0,00 \\
\hline \multirow{4}{*}{$\begin{array}{l}\text { Agachar } \\
\text { (5 pontos) }\end{array}$} & Sem problemas (5) & 13 & 76,47 \\
\hline & Leve dificuldade (4) & 4 & 23,53 \\
\hline & Somente até 90 graus (2) & & 0,00 \\
\hline & Impossível (0) & & 0,00 \\
\hline
\end{tabular}

Fonte: Núcleo de Ortopedia e Traumatologia de Belo Horizonte.

Tabela 3 - Resultados da escala de avaliação do HSS.

FORMULÁRIO DE CLASSIFICAÇÃO DE LIGAMENTO DO JOELHO DO HOSPITAL DE CIRURGIAS ESPECIALIZADAS

THE HOSPITAL FOR SPECIAL SURGERY KNEE LIGAMENT RATING FORM

\begin{tabular}{l|l|c|c}
\hline \multicolumn{1}{c|}{ Perguntas } & \multicolumn{1}{|c}{ Respostas } & $\begin{array}{c}\text { Valor } \\
\text { absoluto }\end{array}$ & $\%$ \\
\hline Sintomas (5 pontos) & \multicolumn{3}{|c}{} \\
\hline Edema & Não $=2$ & 17 & 100,00 \\
\hline & Sim $=0$ & & 0,00 \\
\hline Bloqueio & Não $=3$ & 17 & 100,00 \\
\hline \multicolumn{4}{|l|}{} \\
\hline Falseio (20 pontos) & Sim $=0$ & 17 & 0,00 \\
\hline Intensidade & Nenhuma $=10$ & 100,00 \\
\hline & Transitório $=8$ & & 0,00 \\
\hline & Recorrencia $<1$ dia $=6$ & & 0,00 \\
\hline & Recorrencia $<1$ semana $=2$ & 0,00 \\
\hline & Recorrencia $>1$ semana $=0$ & 17 & 0,00 \\
\hline Frequencia & Nenhuma $=10$ & & 0,00 \\
\hline & 1 por ano $=8$ & & 0,00 \\
\hline & $2-6$ por ano $=6$ & & 0,00 \\
\hline & 1 por mês $=4$ & & 0,00 \\
\hline & 1 por semana $=2$ & & 0,00 \\
\hline & Diário $=0$ &
\end{tabular}

\section{FORMULÁRIO DE CLASSIFICAÇÃO DE LIGAMENTO DO JOELHO DO HOSPITAL} DE CIRURGIAS ESPECIALIZADAS

THE HOSPITAL FOR SPECIAL SURGERY KNEE LIGAMENT RATING FORM

\begin{tabular}{l|l|c|c}
\hline \multicolumn{1}{c|}{ Perguntas } & \multicolumn{1}{|c|}{ Respostas } & $\begin{array}{c}\text { Valor } \\
\text { absoluto }\end{array}$ & $\%$ \\
\hline Função (20 pontos) & Retorno total = 4 & 17 & 100,00 \\
\hline Trabalho & Limitado ou mudança de trabalho $=2$ & & 0,00 \\
\hline & Incapaz devido ao joelho $=0$ & & 0,00 \\
\hline Esporte & Retorno total = 4 & 15 & 88,24 \\
\hline & Retorno com modificação $=3$ & 1 & 5,88 \\
\hline & Mudança de esporte = & 1 & 5,88 \\
\hline & Sem retorno = 0 & & 0,00 \\
\hline Habilidade para & Desacelerar = 4 & 17 & 100,00 \\
\hline & Correr de um lado para o outro =4 & 17 & 100,00 \\
\hline & Pular =4 & 17 & 100,00 \\
\hline
\end{tabular}

\section{Exame (45 pontos) \\ ADM}

1501088,24

Derrame

\section{Circunferencia da coxa}

Lachman (end point)



\section{Gaveta anterior}

\begin{tabular}{l|l} 
Normal $=3$ \\
\hline Flexão ou extenção limitadas $=1$ \\
Ambas $=0$ \\
Não $=4$ \\
Sim $=0$
\end{tabular}

\begin{tabular}{l|l|}
\hline & $1+=2$ \\
\hline & $2+=0$ \\
\hline & $3+=0$ \\
\hline Gaveta posterior & Negativo $=5$ \\
\hline & $1+=3$ \\
\hline & $2+=2$ \\
\hline Pivot Shift & $3+=0$ \\
\hline & Negativo ou simétrico $=10$ \\
\hline & Leve, sem movimento $=8$ \\
\hline & $1+$, movimento leve $=4$ \\
\hline & $2+$, movimento definido $=2$ \\
\hline LCM & $3+$, movimento e bloqueio $=0$ \\
\hline & Normal $=5$ \\
\hline & $1+=3$ \\
\hline & $2+=2$ \\
\hline LCL & $3+=0$ \\
\hline & Normal $=5$ \\
\hline & $1+=3$ \\
\hline Pivot Shift reverso & $2+=2$ \\
\hline & $3+=0$ \\
\hline Exam & Negativo $=5$ \\
\hline & Positivo $=0$ \\
\hline
\end{tabular}

Exame funcional (10 pontos)

Igual ou com discrepancia $<1 \mathrm{~cm}=2$ Discrepancia $>1 \mathrm{~cm}=0$

\begin{tabular}{l|l|c|c}
\hline $\begin{array}{l}\text { Pular em um membro } \\
\text { diferença entre os MMII }\end{array}$ & $90-100 \%=10$ & 14 & 82,35 \\
\hline & $75-90 \%=7$ & 3 & 17,65 \\
\hline & $50-75 \%=5$ & & 0,00 \\
\hline & $50 \%=0$ & & 0,00 \\
\hline
\end{tabular}

\section{Deduções}

\begin{tabular}{|l|c|c|}
\hline Negativo $=4$ & 13 & 76,47 \\
\hline $1+=3$ & 4 & 23,53 \\
\hline $2+=2$
\end{tabular}

\begin{tabular}{l|l|c|c}
\hline Suporte externo & Por insegurança = 2 & & 0,00 \\
\hline & Por instabilidade = 4 & & 0,00 \\
\hline Dor & Nenhuma = & & 0,00 \\
\hline & Ocasional = 2 & 2 & 11,76 \\
\hline & Após esporte severo = 5 & 2 & 11,76 \\
\hline & Após atividades diárias = 8 & & 0,00 \\
\hline & Contínua = 10 & & 0,00 \\
\hline
\end{tabular}


Tabela 4 - Evolução dos pacientes de acordo com as escalas de avaliação.

\begin{tabular}{|c|c|c|c|c|}
\hline \multicolumn{5}{|c|}{ Resumo da Avaliação Clínica } \\
\hline & \multicolumn{2}{|c|}{ Ano 1} & \multicolumn{2}{|c|}{ Ano 10} \\
\hline Pacientes & Score Lysholm & HSS & Lysholm & HSS \\
\hline 1 & 100 & 98 & 100 & 98 \\
\hline 2 & 100 & 96 & 100 & 96 \\
\hline 3 & 100 & 98 & 100 & 98 \\
\hline 4 & 100 & 96 & 100 & 96 \\
\hline 5 & 95 & 96 & 94 & 90 \\
\hline 6 & 95 & 93 & 91 & 93 \\
\hline 7 & 100 & 92 & 95 & 92 \\
\hline 8 & 99 & 98 & 99 & 98 \\
\hline 9 & 99 & 93 & 95 & 87 \\
\hline 10 & 91 & 78 & 86 & 87 \\
\hline 11 & 100 & 98 & 100 & 98 \\
\hline 12 & 100 & 100 & 100 & 100 \\
\hline 13 & 99 & 97 & 95 & 97 \\
\hline 14 & 100 & 98 & 100 & 98 \\
\hline 15 & 100 & 98 & 100 & 98 \\
\hline 16 & 100 & 98 & 100 & 98 \\
\hline 17 & 100 & 95 & 100 & 95 \\
\hline Média & 98,71 & 95,41 & 97,35 & 95,24 \\
\hline Desvio Padrão & 2,57 & 4,99 & 4,11 & 4,02 \\
\hline Variancia & 6,48 & 24,87 & 16,40 & 16,19 \\
\hline
\end{tabular}

Fonte: Núcleo de Ortopedia e Traumatologia de Belo Horizonte.

Nenhum paciente se queixou de dor na área doadora do enxerto após quatro semanas da cirurgia até a última avaliação. Quatro pacientes sofreram ruptura do LCA contralateral em atividades esportivas, sendo três entorses em futebol (ocorridas três, quatro e dez anos após a cirurgia) e uma durante dança, ocorrida três anos após o procedimento.

Três pacientes apresentavam no exame radiológico alguma diminuição do espaço articular femorotibial medial. Esses pacientes foram submetidos a meniscectomias. Não houve diminuição de espaço articular femoropatelar, com exceção de um paciente que sofreu fratura da patela seis meses depois da cirurgia após cair de escada (Figura 4).

\section{DISCUSSÃO}

A reconstrução do LCA tem como objetivo estabilizar o joelho. A restauração da cinemática minimiza as forças anormais na interface femorotibial. Episódios recorrentes de instabilidade após a ruptura do LCA estão frequentemente associados a lesões de menisco e osteoartrose prematura. Artroscopias realizadas em pacientes com lesões antigas e não tratadas do LCA apresentam grande número de lesões meniscais e condrais. Essas articulações têm grande incidência de osteoartrose, mesmo após a reconstrução ${ }^{(14,15)}$.

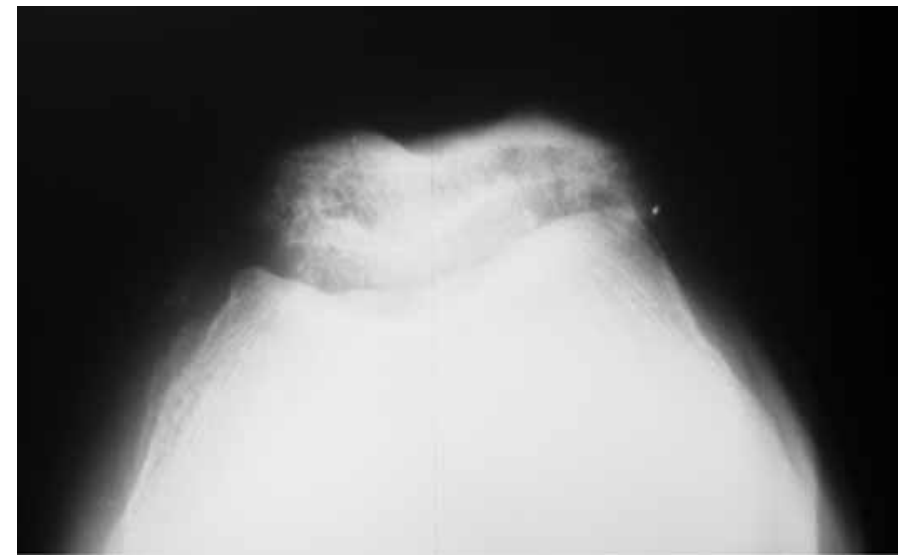

Figura 4 - Radiografia em axial de patela em $30^{\circ}$ de flexão do joelho após reconstrução do LCA em paciente que sofreu fratura da patela seis meses após a cirurgia, submetido a osteossíntese com banda de tensão já retirada

O tendão do quadríceps é parte integrante do aparato extensor do joelho. Cobre a tróclea femoral à medida que o joelho é dobrado. É formado por múltiplas camadas de colágeno que variam em forma e tamanho. Essas camadas se sobrepõem de forma oblíqua e longitudinal, formando complexa banda de tensão. A parte mais anterior do músculo reto femoral cria uma aponeurose na frente da patela, que é chamada de retináculo pré-patelar ${ }^{(14,16)}$. A parte mais posterior do TQ funde-se com a parte mais anterior da membrana sinovial. A gordura suprapatelar cobre sua parte distal.

Harris et $a l^{(17)}$ demonstraram que, em média, o TQ é 1,8 vez mais espesso que o TP. À distância de 7 a 10mm entre o tendão e a cartilagem articular, cria uma área segura para a retirada do enxerto sem trauma da superfície articular da patela. O TQ é suficientemente largo (média de $27 \mathrm{~mm}$ ) para a retirada de enxertos de $10 \mathrm{~mm}$ de diâmetro. Apresenta ainda inserção assimétrica com discreto desvio lateral de suas fibras. Sugere-se que a retirada do enxerto deve seguir esse sentido ${ }^{(17)}$.

Tomando-se por base uma área central do TQ e TP de $10 \mathrm{~mm}$, os estudos de Staubli e Jakob ${ }^{(11)}$ revelam que o TQ apresenta comprimento entre $64,4 \mathrm{~mm}$ e $61,9 \mathrm{~mm}$ de acordo com o tipo de preparo e forças que agem sobre a peça. $\mathrm{O}$ TP apresenta comprimento entre $36,8 \mathrm{~mm}$ e $34,5 \mathrm{~mm}$, nas mesmas condições de pesquisa. Notaram que o TQ apresenta uma área ampla de fixação na patela. As fibras mais anteriores do TQ avançam anteriormente à patela, formando aponeurose que cria um sistema de banda de tensão. A aponeurose funde-se com as fibras mais anteriores do TP. Nesses cortes os mesmos autores mostraram que a junção miotendinosa do TQ está localizada $85 \mathrm{~mm}$ proximal à base da patela. Em cortes do TP chamaram a atenção para a pequena área de inserção do TP na patela em relação ao TQ. 
Segundo Staubli e Jakob ${ }^{(11)}$, a análise da força tênsil de enxertos de $10 \mathrm{~mm}$ de espessura retirados da parte central do TQ e TP de adultos jovens (média de idade de 24,9 anos, variando entre 19 e 32 anos) revelou que o ponto de ruptura para tendões sem preparo prévio é de $2.173 \mathrm{~N}( \pm 618 \mathrm{~N})$ para o TQ e de $1.953 \mathrm{~N}( \pm 325 \mathrm{~N})$ para o TP. Nos estudos de ciclos de resistência, quando submetidos a 200 ciclos de 50 a $800 \mathrm{~N}$, com frequência de $0,5 \mathrm{~Hz}$, o ponto de falência do TQ é de $2.353 \mathrm{~N}( \pm 495 \mathrm{~N})$ e de $2.376 \mathrm{~N}( \pm 152 \mathrm{~N})$ para o TP. Apesar de o tamanho, espessura, forma e área do TQ e TP serem diferentes no início dos testes, os pontos de ruptura e a energia necessária para falência não foram significativamente diferentes. A diferença de $200 \mathrm{~N}$ entre os resultados não possui relevância clínica. Harris et al ${ }^{(17)}$, em seu trabalho sobre resistência do TQ, mostraram que a falência da sutura ocorria a $692 \mathrm{~N}( \pm 181 \mathrm{~N})$ e que a falência do tendão ocorria a $1.075 \mathrm{~N}( \pm 449 \mathrm{~N})$. Esse ponto de ruptura era 1,36 vez maior no TQ quando comparado com o TP.

$\mathrm{O}$ desconforto na região anterior do joelho após a reconstrução da LCA permanece insolúvel. Muitas causas têm sido propostas, incluindo limitações da ADM após a cirurgia, condromalácia, programa de reabilitação inadequado, dor no sítio doador de enxerto, presença de contratura fixa, redução do tamanho do TP, patela baixa, alterações no glucamato e substância-P(18-21). Deehan et $a l^{(16)}$ encontraram baixa incidência $(8 \%)$ de crepitação na articulação femoropatelar, após a reconstrução do LCA com tendão patelar. McDaniel e Dameron ${ }^{(22)}$ encontraram $45 \%$. Deehan et al ${ }^{(16)}$ também relatam que, após cinco anos de seguimento, 91\% de seus pacientes não apresentavam dor ou tinham dor leve no tendão patelar. Dor no agachamento era problema persistente, $44 \%$ dos pacientes descrevendo desconforto ao ajoelhar após cinco anos de cirurgia. Nos pacientes desse estudo não houve dor na área doadora do enxerto. A tendinite patelar foi rara nos primeiros seis meses de PO e apresentou rápida resolução com exercícios de alongamento.

Por ser técnica pouco usada no passado recente, existem poucos trabalhos de avaliação de longo prazo. Chen et al $^{(23)}$ avaliaram 34 pacientes com seguimento variando entre quatro e sete anos, com média de 62 meses. Houve $94 \%$ de bons e excelentes resultados avaliados pela escala de Lysholm. O retorno ao esporte moderado ou extremo ocorreu em $76 \%$ dos pacientes. Observaram-se 88 pacientes com frouxidão ligamentar menor que $2 \mathrm{~mm}$. Usando a escala IKDC, 91\% dos pacientes apresentavam joelho normal ou perto do normal. Concluem que o TQ é boa escolha para a reconstrução do LCA.
Lee et al $^{(24)}$ avaliaram clinicamente 67 pacientes com média de seguimento de 41 meses. Usando a escala de Lysholm, pacientes progrediram de 71 pontos no préoperatório para 90 no PO $(\mathrm{P}<0,05)$. Na escala IKDC, $94 \%$ dos pacientes eram grau A e B. Na avaliação isocinética, o pico de torque na extensão foi de $82 \%$ e $89 \%$ comparado com o outro joelho, em um e dois anos de pós-operatório, respectivamente. Não houve alteração nos índices de Insall-Salvati e congruência patelar.

Joseph et $a l^{(25)}$ compararam três diferentes enxertos para reconstrução do LCA. Relataram que os pacientes do grupo do TQ necessitaram menor quantidade de analgésicos e recuperaram a extensão ativa mais rapidamente que o grupo em que foi usado o ligamento patelar.

Em nosso meio, Coelho et $a^{(26)}$, Cortellazo et $a^{(27)} \mathrm{e}$ Guimarães et al ${ }^{(13,28,29)}$ publicaram trabalhos sobre o uso do enxerto do TQ na reconstrução do LCA.

O TQ tem sido usado com sucesso para revisão de cirurgia de reconstrução do LCA e na reconstrução do LCA por banda dupla ${ }^{(30-32)}$. Antonogiannakis et al ${ }^{(33)}$ mostraram estudo com o TQ em cirurgia para reconstrução do LCA sem o bloco ósseo da patela, com bons resultados.

Pigozzi et al ${ }^{(34)}$ compararam a evolução isocinética de pacientes operados com LP e TQ. Concluíram que o déficit de força no grupo do TQ era menor e que a boa recuperação de força nesse grupo após seis meses de PO deveria encorajar o uso do TQ nas reconstruções do LCA.

Um dos indicadores de sucesso de reconstrução de LCA é a taxa de retorno ao esporte. Tal evento pode ser influenciado pelo estado pré-operatório do indivíduo, expectativa, segurança do paciente, reabilitação e o tipo do esporte. Gobbi e Francisco ${ }^{(35)}$ avaliaram 100 atletas submetidos à reconstrução do LCA com TP $(n=50)$ e tendões flexores $(n=50)$. Observaram que $64 \%$ retornaram ao mesmo esporte no mesmo nível, 24\% mudaram de esporte e $11 \%$ interromperam a atividade esportiva. Não houve diferença entre os pacientes dos dois grupos. Nos pacientes desse estudo observa-se maior retorno ao esporte quando comparados com os achados de Gobbi e Francisco ${ }^{(35)}$.

Chama atenção a importância da preservação dos meniscos para os pacientes com lesão ligamentar. Gillquist e Messner ${ }^{(36)}$ afirmaram que lesões concomitantes do LCA e menisco aumentam a incidência de alterações degenerativas no joelho. Cohen et al $^{(37)}$ mostraram a associação ruim entre meniscectomia, pacientes com lesão do LCA operados e artrose em longo prazo. Carvalho Junior et al ${ }^{(38)}$ mostraram os efeitos da meniscectomia nos resultados da reconstrução do LCA. Nesse trabalho, os três pacientes de pior pontuação foram submetidos a meniscectomias totais, uma na cirurgia primária e os outros dois, anos após. 
Observou-se que mesmo com joelho estável, muitos pacientes necessitaram de outras intervenções para correção de lesões de meniscos e/ou condral. Os autores acreditam que as lesões secundárias e associadas à lesão do LCA continuam deteriorando o joelho ao longo do tempo, apesar de a estabilidade ter sido restaurada. Fithian et al ${ }^{(39)}$ mostraram alta incidência de degenerações tardias em pacientes submetidos à reconstrução do LCA. Seria desejável que os pacientes com importantes lesões meniscais fossem desencorajados a retornar a determinados esportes.

Outro fator importante foi a alta taxa de lesão do LCA contralateral observada (17,65\%). Existem várias teorias para explicar esse achado. Uma delas vincula essa observação ao déficit em avaliação isocinética no membro contralateral (não operado), fato já observado por Guimarães et $a l^{(28)}$.

\section{REFERÊNCIAS}

1. Wetzler MJ, Bartolozzi AR, Gillespie MJ. Revision anterior cruciate ligament reconstruction. Oper Tech Orthop. 1996;6:181-9.

2. Sachs RA, Daniel DM, Stone ML, Garfein RF. Patellofemoral problems after anterior cruciate ligament reconstruction. Am J Sports Med. 1989;17(6): 760-5.

3. Langan P, Fontanetta AP. Rupture of patellar tendon after use of its central third. Orthop Rev. 1987;16(3):317-21

4. Rosenberg TD, Franklin JL, Baldwin GN, Nelson KA. Extensor mechanism function after patellar tendon graft harvest for anterior cruciate ligament reconstruction. Am J Sports Med. 1992;20(5):519-25.

5. Cho KO. Reconstruction of the anterior cruciate ligament by semitendinosus tenodesis. J Bone Joint Surg Am. 1975;57:608-12.

6. Eriksson K, Anderberg P, Hamberg P, Lofgren AC, Bredenberg M, Westman I, Wredmark T. A comparison of quadruple semitendinosus and patellar tendon grafts in reconstruction of anterior cruciate ligament. J Bone Joint Surg. 2001;83:348-54.

7. Marshall JL, Warren RF, Wickiewicz TL, Reider B. The anterior cruciate ligament: a technique for repair and reconstruction. Clin Orthop Relat Res. 1979;(143): 97-106.

8. Fulkerson JP, Langeland R. An alternative cruciate reconstruction graft: the central quadriceps tendon. Arthroscopy. 1995;11(2):252-4

9. Howe JG, Johnson RJ, Kaplan MJ, Fleming B, Jarvinen M. Anterior cruciate ligament reconstruction using quadriceps patellar tendon graft. Part I. Long-term followup. Am J Sports Med. 1991;19(5):447-57.

10. Staubli HU, Schatzmann L, Brunner P, Rincon L, Nolte LP. Quadriceps tendon and patellar ligament: cryosectional anatomy and structural properties in young adults. Knee Surg Sports Traumatol Arthrosc. 1996;4(2):100-10.

11. Staubli HU, Jakob RP. Central quadriceps tendon for anterior cruciate ligament reconstruction. Part I: morphometric and biochemical evaluation. Am J Sports Med. 1997;25(5):725-7.

12. Almazan Diaz A, Cruz Lopez F, Perez Jimenez FX, Ibarra Ponce de Leon JC. Minimally invasive quadriceps tendon harvest. Arthroscopy. 2006;22(6):679.e1-3.

13. Guimaraes MV. Reconstrução artroscópica do ligamento cruzado anterior: estudo comparativo entre os enxertos de ligamento patelar e de tendão do quadríceps. Rev Bras Ortop. 2004;39(2):130-41

14. Jomha NM, Borton DC, Clingeleffer AJ, Pinczewski LA. Long-term osteoarthritic changes in anterior cruciate ligament reconstructed knees. Clin Orthop Relat Res. 1999;(358):188-93.

15. Dandy DJ, Hobby JL. Anterior cruciate ligament reconstruction. J Bone Joint Surg Br. 1998;80(2):189-90.

16. Deehan DJ, Salmon LJ, Webb VJ, Davies A, Pinczewski LA. Endoscopic reconstruction of the anterior cruciate ligament with an ipsilateral patellar tendon autograft. $A$ prospective longitudinal five-year study. J Bone Joint Surg Br. 2000;82(7):984-91.

17. Harris NL, Smith DA, Lamoreaux L, Purnell M. Central quadriceps tendon for anterior cruciate ligament reconstruction. Part I: Morphometric and biomechanical evaluation. Am J Sports Med. 1997;25(1):23-8.

18. Aglietti P, Buzzi R, D'Andria S, Zaccherotti G. Patellofemoral problems after intraarticular anterior cruciate ligament reconstruction. Clin Orthop Relat Res. 1993;(288):195-204.

19. Shino K, Nakagawa S, Inoue M, Horibe S, Yoneda M. Deterioration of patellofemora articular surfaces after anterior cruciate ligament reconstruction. Am J Sports Med. 1993;21(2):206-11

20. Hantes ME, Zachos VC, Bargiotas KA, Basdekis GK, Karantanas AH, Malizos KN Patellar tendon length after anterior cruciate ligament reconstruction: a comparative magnetic resonance imaging study between patellar and hamstring tendon autografts. Knee Surg Sports Traumatol Arthrosc. 2007;15(6):712-9.
Este artigo tem limitações. O autor principal tem como primeira opção o uso do TQ na reconstrução do LCA. Isso inviabilizou um estudo comparativo entre dois tipos diferentes de enxertos. O número de pacientes estudado é pequeno. Avaliação anual seria importante para dar detalhes da evolução dos pacientes. Faltam dados clínicos dos pacientes no pré-operatório, perdidos no arquivo.

\section{CONCLUSÃO}

O enxerto do TQ é boa opção para a reconstrução do LCA, mesmo quando decorridos 10 anos do procedimento cirúrgico. Não houve dor na área doadora do enxerto em médio e longo prazo. A taxa de retorno ao esporte foi excelente e não houve alteração da articulação femoropatelar.

21. Witonski D, Wagrowska-Danielewicz M. Distribution of substance-P nerve fibers in the knee joint in patients with anterior knee pain syndrome. A preliminary report. Knee Surg Sports Traumatol Arthrosc. 1999;7(3):177-83.

22. McDaniel WJ Jr, Dameron TB Jr. Untreated ruptures of the anterior cruciate ligament. A follow-up study. J Bone Joint Surg Am. 1980;62(5):696-705.

23. Chen $\mathrm{CH}$, Chuang TY, Wang KC, Chen WJ, Shih $\mathrm{CH}$. Arthroscopic anterior cruciate ligament reconstruction with quadriceps tendon autograft: clinical outcome in 4-7 years. Knee Surg Sports Traumatol Arthrosc. 2006;14(11):1077-85.

24. Lee S, Seong SC, Jo H, Park YK, Lee MC. Outcome of anterior cruciate ligament reconstruction using quadriceps tendon autograft. Arthroscopy. 2004;20(8): 795-802.

25. Joseph M, Fulkerson J, Nissen C, Sheehan TJ. Short-term recovery after anterior cruciate ligament reconstruction: a prospective comparison of three autografts. Orthopedics. 2006;29(3):243-8.

26. Coelho MG, Souza JMG, Caldas MTL. Reconstrução "all-inside" do ligamento cruzado anterior do joelho com enxerto da parte central do tendão quadricipital. Descrição técnica. Rev Bras Ortop. 1998;33(5):357-62

27. Cortelazo MJ, Cohen M, Mestriner LA, Carneiro FM. Reconstrução artroscópica do ligamento cruzado anterior com enxerto do tendão quadricipital: estudo das características dimensionais do tendão e da técnica. Nota preliminar. Rev Bras Ortop. 2002;37(6):247-55.

28. Guimarães MV, Cunha FM, Silva WN. Reconstrução do ligamento cruzado anterior utilizando enxerto de tendão do quadríceps: avaliação clínica e isocinética. Rev Bras Ortop. 2005;40(4):182-92.

29. Guimaraes MV, Murta Filho JB, Baumfeld DS, Da Silva WN. Ensanchamiento del tunel tibial tras reconstruir LCA con injerto de tendon cuadriceps: comparacion entre tres tecnicas de fijacion. Artrosc. (B. Aires). 2007;14(2):111-8.

30. Garofalo R, Djahangiri A, Siegrist $O$. Revision anterior cruciate ligament reconstruction with quadriceps tendon-patellar bone autograft. Arthroscopy. 2006;22(2):205-14.

31. Noronha JC. Reconstruction of the anterior cruciate ligament with quadriceps tendon. Arthroscopy. 2002;18(7):E37.

32. Kim SJ, Jung KA, Song DH. Arthroscopic double-bundle anterior cruciate ligament reconstruction using autogenous quadriceps tendon. Arthroscopy. 2006;22(7): 797.e1-5.

33. Antonogiannakis E, Yiannakopoulos CK, Hiotis I, Karabalis C, Babalis G. Arthroscopic anterior cruciate ligament reconstruction using quadriceps tendon autograft and bioabsorbable cross-pin fixation. Arthroscopy. 2005;21(7):894.

34. Pigozzi F, Di Salvo V, Parisi A, Giombini A, Fagnani F, Magini W, et al. Isokinetic evaluation of anterior cruciate ligament reconstruction: quadriceps tendon versus patellar tendon. J Sports Med Phys Fitness. 2004;44(3):288-93.

35. Gobbi A, Francisco R. Factors affecting return to sports after anterior cruciate ligament reconstruction with patellar tendon and hamstring graft: a prospective clinical investigation. Knee Surg Sports Traumatol Arthrosc. 2006;14(10):1021-8.

36. Gillquist J, Messner K. Anterior cruciate ligament reconstruction and the long-term incidence of gonarthrosis. Sports Med. 1999;27(3):143-56.

37. Cohen M, Amaro JT, Ejnisman B, Carvalho RT, Nakano KK, Peccin MS, et al. Anterior cruciate ligament reconstruction after 10 to 15 years: association between meniscectomy and osteoarthrosis. Arthroscopy. 2007;23(6):629-34.

38. Carvalho Junior LH, Pidner Neto H, Schuffner FA. Efeitos da meniscectomia no resultado da reconstrução do ligamento cruzado anterior. Rev Bras Ortop. 2004;39(7):357-62.

39. Fithian DC, Paxton EW, Stone ML, Luetzow WF, Csintalan RP, Phelan D, et al. Prospective trial of a treatment algorithm for the management of the anterior cruciate ligament-injured knee. Am J Sports Med. 2005;33(3):335-46. 